\title{
Des fonctions économiques des tribunaux
}

\section{Evelyne Serverin}

\section{(2) OpenEdition}

Journals

Édition électronique

URL : http://journals.openedition.org/ei/811

DOI : 10.4000/ei.811

ISSN : 2553-1891

\section{Éditeur}

Association Économie et Institutions

\section{Édition imprimée}

Date de publication : 1 juin 2004

Pagination : 97-127

ISSN : 1775-2329

\section{Référence électronique}

Evelyne Serverin, "Des fonctions économiques des tribunaux », Économie et institutions [En ligne],

4 | 2004, mis en ligne le 31 janvier 2013, consulté le 01 mai 2019. URL : http://

journals.openedition.org/ei/811 ; DOI : 10.4000/ei.811 


\section{Des fonctions économiques des tribunaux}

\section{Evelyne Serverin ${ }^{1}$}

Sur fond d'un intérêt croissant des sciences sociales, et notamment de l'économie, pour la justice (1 ère partie), nous proposons d'aborder le tribunal sous l'angle de la fonction économique qui lui est attribuée par les règles de droit, situées au plan de son organisation (2ème partie), comme de son accessibilité (3ème partie).

Par fonction économique, nous entendons la garantie apportée par les tribunaux ordinaires aux échanges, l'échange constituant le point de liaison entre le droit et l'économie (Commons, 1938, p. 129, Serverin, 2001, p.43). Cette définition met au premier plan le système juridictionnel de droit commun, et exclut les actions et juridictions spécialisées qui relèvent de ce qui est communément reconnu comme «droit économique» (Kirat et Serverin, 2000, p.11)2. En tant que cette fonction économique est abordée sous l'angle des règles de droit, notre démarche sera celle d'un juriste observateur de son système, qui prend le droit comme le «symbole visible d'un certain état social » (Durkheim, [1893], 1978, p.28)33, sans référence aux pratiques. Enfin, en tant que ce propos s'adresse à des économistes, pour qui le comportement des agents est un référentiel obligé, il s'attachera à identifier les institutions juridiques qui peuvent avoir une portée explicative des activités humaines (Ripert, 1951, p. 4)

1 Directeur de recherches au CNRS, IRERP, Institut de recherches sur les relations professionnelles, Université Paris 10 Nanterre.

$2 \mathrm{La}$ fonction économique est attribuée le plus souvent par les juristes comme par les économistes à sa partie la plus visible, celle du droit économique, "qui entend organiser l'enseignement des corps de règles dont l'objet est considéré comme relevant d'une "nature" économique : la concurrence, la distribution, la consommation, la fiscalité, etc. " (Kirat et Serverin, 2000, p. 11).

3 Pour saisir la " solidarité sociale " comme phénomène social, Durkheim se fonde sur les sources juridiques, dont les formes et les transformations constituent la matière quasi exclusive de ses développements. C'est en s'appuyant sur l'observation du droit que Durkheim établira sa célèbre distinction entre les deux formes de solidarité, organique et mécanique.

4 Devant le projet de séparation des filières qui aura lieu en 1954, ce juriste annonçait que les économistes " auraient à se repentir d'avoir ouvert trop facilement les portes de leurs nouvelles facultés à tant d'élèves et quelques maîtres qui prétendraient ne pas avoir à connaître les règles juridiques ", ajoutant "qu'on ne saurait analyser l'activité productrice des hommes si on ne sait pas quelles sont les institutions par lesquelles elles s'exercent ".

95 Economie et Institutions $-n^{\circ} 4-1^{\text {e }}$ semestre 2004 


\section{1- Des sciences sociales divisées sur la fonction des tribunaux}

$\mathrm{Au}$ sein des sciences sociales, la fonction attribuée aux tribunaux dans l'ensemble des relations sociales fait l'objet de lectures contrastées. On tracera les différentes thèses à grands traits, en les situant dans leur dominante disciplinaire, économie, droit et sociologie.

\section{1-1 Rationalités de l'action des tribunaux dans la pensée économique}

Dans leur attention portée au droit, les économistes n'ont nullement méconnu la place des tribunaux, d'autant moins que la plupart ont fait leur première expérience de l'interface entre droit et économie en tant qu'experts dans des procédures judiciaires (Hirsch, 1979, p. 2). Mais selon la valeur accordée à la l'autorité de l'Etat, la fonction attribuée aux tribunaux dans le traitement des litiges sera plus ou moins positivement appréciée.

\section{1-1-1 De l'utilité institutionnelle des tribunaux}

Pour les institutionnalistes, le tribunal joue un rôle important dans la régulation économique, même si les fonctions qui leur sont attribuées ne sont pas identiques.

Commons a principalement mis l'accent sur ce qu'on appellerait la fonction « jurisprudentielle » des tribunaux, en tant qu'ils constituent un lieu de reconnaissance de règles. En effet, dans les transactions entre parties égales, le tribunal est un opérateur central de règlement des conflits d'intérêts (Commons, 1938, p. 132-133). Selon Commons, le tribunal permet la transformation des coutumes en "règles opératoires " pour la période et dans le secteur considérés. (Commons, 1931, p. 651). La connaissance des décisions devient dès lors indispensable pour connaître ces «règles opératoires » qui forment une des catégories de l'action collective.

Les développements contemporains de l'économie institutionnelle du droit se fondent sur l'idée que l'ordre social est le produit de processus politiques et juridiques dans lesquels des droits et obligations réciproques entre individus sont créés et mis en œuvre (Bazzoli et Kirat, 1997). Les tenants de l'institutionnalisme considèrent ainsi que les droits sont des intérêts juridiquement protégés, attirant ainsi l'attention sur les processus, notamment judiciaires, par lesquels les intérêts se voient reconnaître une garantie (Kirat, 1999, p. 15). 
De leur côté, les historiens de l'économie sont de plus en plus attentifs au rôle des tribunaux «au quotidien ». Une spécialiste de l'histoire russe a ainsi pu montrer que l'accroissement constant des litiges dans les tribunaux ruraux par les paysans russes à la veille de la révolution était lié au développement de l'économie de marché (Burbank, 2003, p. 46). Plus récemment, s'est développé un courant d'histoire économique qui aborde les situations de production à partir des contentieux qu'elles suscitent. Il a été ainsi montré, dans le domaine de la qualité des produits, que la construction de la qualité est un processus à la fois technique, économique et institutionnel. La qualité ne doit pas être saisie comme un développement «naturel», qui serait lié à la nature des choses, mais ne peut s'expliquer qu'en prenant en considération «l'interaction entre activités économiques et règles de droit », et notamment dans le contexte «de leur application dans les contentieux » (Stanziani, 2003, p. 73).

L'intérêt porté par cette partie de l'économie aux contentieux appelle la production de connaissances spécifiques sur les procédures, ouvrant la voie à une collaboration renouvelée avec les juristes.

\section{1-1-2 ...à son inefficience dans l'allocation des droits}

A l'opposé, l'analyse économique du droit (Law and Economics), aborde les fonctions des tribunaux d'un point de vue critique.

Dans son article de référence, The Problem of Social Cost, Coase a multiplié les exemples de conflits sur l'attribution des droits en matière de nuisances, et s'est attaché à étudier les précédents issus des tribunaux. Mais contrairement aux institutionnalistes, il tend à contester l'efficience de l'action des tribunaux dans l'allocation des droits. Pour lui, les agents sont $a$ priori les mieux à mêmes de trouver des arrangements efficients, l'intervention du tribunal ne se justifiant que lorsque des arrangements privés sont trop coûteux (Coase, 1960) ${ }^{5}$. Une alternative est ainsi posée entre deux modes de règlement des litiges: le jugement ou l'arrangement direct entre les parties. Plus qu'à une économie centrée sur les règles et les précédents, l'approche coasienne a ouvert la voie à une théorie de la négociation et des arrangements sur les droits.

Cette dernière orientation qui sert aujourd'hui de point d'appui à un corpus important de travaux néo-classiques sur la justice, nord-américains et

5 Pour Coase, "le raisonnement employé par les tribunaux dans la détermination des droits était souvent étrange pour un économiste, parce que nombre de facteurs sur lesquels reposent les décisions sont, pour un économiste, irrelevants" (Coase, 1960, p. 15).

97 Economie et Institutions $-\mathrm{n}^{\circ} 4-1^{\mathrm{e}}$ semestre 2004 
européens, qui appliquent aux tribunaux leur critère habituel d'efficience. Les travaux pionniers de Landes (1971), suivis par Landes et Posner (1979), affirment ainsi que la fonction de règlement des conflits pourrait être plus efficacement assurée par un marché privé et concurrentiel des services de justice (l'arbitrage), que par des tribunaux financés sur budget de l'Etat. Dans cette analyse, le contentieux ordinaire est dissocié de la production du précédent, à laquelle est accordée au contraire une valeur économique, selon une conception proche de celle de Commons (Landes et Posner, 1976, p. 202).

Dans leur sillage, les microéconomistes de la justice, principalement nord-américains, partagent la conviction que le recours aux tribunaux est une solution non coopérative, et tentent de modéliser les motivations individuelles du recours à la justice en vue de définir les politiques qui permettraient de développer les règlements amiables. Ces modèles peuvent être grossièrement classés selon qu'ils se situent antérieurement ou postérieurement à l'action en justice, les premiers étudient les circonstances qui contribuent à influencer la décision d'aller au tribunal, les seconds étudiant les circonstances de l'abandon des procédures (Doriat-Duban, 2001, p. 184). Mais tous sont orientés vers une interprétation négative de l'intervention du tribunal dans le règlement final du litige.

Sans vouloir discuter ici la pertinence de ces modèles du point de vue des comportements, on retiendra qu'ils ne laissent place à aucune interrogation sur les fonctions assurées par les tribunaux, et se désintéressent donc des procédures qui les organisent et des actions qui y sont portées.

\section{1-2 Théories juridiques de la fonction décisionnelle des tribunaux}

Le statut donné aux tribunaux n'est pas non plus uniforme en théorie du droit. Pour faire bref, et en nous en tenant aux modèles théoriques continentaux, on opposera la conception normativiste, qui reconnaît aux tribunaux une fonction seulement sanctionnatrice, à l'analyste réaliste, qui retient une fonction proprement créative du droit des tribunaux.

\section{1-2-1 Le tribunal sanctionnateur dans les théories normatives}

La conception kelsénienne de la science du droit, dominante en France, conduit à mettre en avant une définition de la norme comme commandement adressé aux sujets, et assorti de sanctions (Kelsen, 1959). Dans un énoncé du type « le vol est puni d'emprisonnement », Kelsen (et à travers lui, le juriste qui décrit les normes), lit deux prescriptions: la première est une prescription d'appliquer la sanction, et elle s'adresse aux 
autorités ; la seconde est une prescription de se comporter comme la norme l'indique, et elle s'adresse aux sujets de l'ordre juridique. Si les deux catégories de prescriptions sont soigneusement distinguées, elles ne sont pas indépendantes, et il est clair que la seconde est plus importante que la première. Cette hiérarchie est particulièrement visible lorsque Kelsen définit le critère d'efficacité de l'ordre juridique : « une norme juridique, c'est-à-dire une norme qui prescrit que, sous certaines conditions, en particulier sous la condition que les hommes se comportent d'une certaine manière, un acte contraignant, en guise de sanction, doit être dirigé vers ces hommes [...], est efficace si elle est suivie, c'est-à-dire si les sujets de l'ordre juridique manifestent un comportement qui évite la sanction; ou si, dans le cas d'un comportement conditionnant la sanction, la norme juridique est appliquée, c'est-à-dire si la sanction prescrite par la norme juridique est effectivement ordonnée et exécutée par les organes compétents » (Kelsen, 2000, p.25. Du point de vue logique, la norme constitue une variante d'impératif, appelant obéissance de la part des assujettis. Le but du droit, comme l'indique Kelsen, n'est rien d'autre que « la prévention du comportement illégal, c'est-à-dire du comportement conditionnant la sanction » (Kelsen, 2000, pp. 22-23).

La constante référence à la fonction sanctionnatrice du tribunal est caractéristique de la position kelsénienne, et se trouve largement partagée par la pensée juridique continentale. Cette analyse a pour conséquence d'assujettir le procès au droit substantiel, le premier n'étant que le bras armé du second, sans laisser place à une autonomie de la fonction juridictionnelle.

\section{1-2-2 Le tribunal créateur de règles dans les théories analytiques}

Les juristes réalistes, notamment scandinaves, ont donné une toute autre place au juge. S'il n'est pas nié que le droit soit composé de normes, ni que le contenu d'une norme juridique soit une prescription d'adopter un comportement donné, une norme ne sera dite valide que si le juge l'applique. La théorie réaliste s'attache donc à étudier les conditions de la prise de décision, en observant la manière dont le juge applique les directives, et en évaluant notamment le poids de ces directives parmi les autres facteurs intervenant dans la prise de décision: « la question de savoir dans quels rapports se trouvent, en réalité, le texte fixé et les motifs libres dans l'interprétation (...), ne peut naturellement être résolue d'une manière générale, mais seulement par l'analyse des méthodes qui, actuellement, gouvernent la juridiction dans un lieu et un moment donné » (Ross, 1957). D'où l'importance des énoncés prédictifs du comportement futur des juges. En dernière analyse, dire qu'une règle $D$ est valide revient à énoncer une proposition prédictive que les tribunaux, dans certaines circonstances, fonderont leurs décisions sur la directive $D$. La « validité » du droit n'est pas donnée a priori, mais découverte par la recherche.

99 Economie et Institutions $-n^{\circ} 4-1^{\text {e }}$ semestre 2004 
La théorie du droit de Herbert Hart, fondée sur la démarche analytique, s'appuie sur cette même distinction entre les destinataires de la règle. Hart oppose ainsi les règles primaires et les règles secondaires, les premières visant les actions des simples citoyens, les secondes conférant des pouvoirs destinés à parachever les effets juridiques des règles primaires. Ce qui fait droit dans les sociétés évoluées est l'union de règles primaires et secondaires. Parmi ces règles secondaires figurent notamment celles qui habilitent les juges «à résoudre d'autorité la question de savoir si, en des circonstances particulières, les règles primaires ont été transgressées» (Hart, 1976, p.122). Le juge se voit habilité à produire des «règles de décision» (rules of adjudication) qui présentent une double particularité: elles constituent d'une part des «actes attributifs» de droits subjectifs et de responsabilité ; elles constituent une source du droit, moyennant une inférence, certes «quelque peu hasardeuse», dont la sûreté «varie à la fois en fonction de l'habileté de l'interprète et de l'esprit de suite des juges» (Hart, 1976, p.123).

La démarche de H.L.A. Hart le conduit ainsi à définir un véritable programme de recherche juridique analytique non empirique: ce programme implique «de prêter à l'analyse des concepts juridiques et politiques une attention plus minutieuse que celle qu'on lui accorde habituellement», et de procéder à cette analyse, non dans «les termes d'un discours ordinaire ou scientifique de constatation ou de prédiction», mais de manière « interne au système juridique».

L'attention portée à la forme des règles qui s'adressent aux juges constitue une première voie $\mathrm{d}^{\prime}$ accès aux fonctions du tribunal. Sans ouvrir à une observation des pratiques des tribunaux (programme qui relève la sociologie du droit), elle permet d'en établir le cadre et d'en comprendre le déroulement.

\section{1-3 Le recours aux tribunaux comme pratique sociale}

La sociologie du droit, qui a en charge la production de connaissances empiriques sur le droit dans la société, n'a pas ignoré le rôle des tribunaux. Et de fait, les programmes et les questionnements sur ce point ont été permanents dans tous les Etats qui connaissent un système judiciaire, même peu développé. Sans pouvoir en tenter fût ce une synthèse, on fera le choix du contraste pour retenir trois écoles : celle qui se préoccupe de l'accès aux tribunaux, celle qui valorise les moyens de leur évitement, et celle qui, sans entrer dans le débat de l'opportunité du recours ou du non recours au tribunal, considère l'appareil de justice comme une variable du calcul économique des agents. 


\section{1-3-1 Les enquêtes sur l'accès aux tribunaux}

Aux Etats-Unis comme en Europe, le milieu du 20ème siècle a vu se développer d'importants programmes d'accès au droit. On peut citer une vaste enquête de droit comparé, menée dans le cadre de l'Institut européen de Florence (connue sous le nom de programme de Florence), qui s'est déroulée tant en Europe qu'aux Etats-Unis à la fin des années soixante-dix. Dans cette étude, l'accès aux droits constituait un objectif, commun à divers procédés : l'aide judiciaire, la protection des intérêts diffus, les règlements hors des tribunaux, et les mesures étatiques visant à permettre l'efficacité des lois. L'aide judiciaire notamment était considérée comme un procédé de «première vague », nécessaire, sans être suffisant, à la réalisation effective des droits.

Aux Etats-Unis, l'intérêt pour l'accès aux tribunaux était plus ancien, et les sociologues se sont associés à des juristes sous l'égide de l'association Law and Society, (créée en 1964), à l'occasion d'un projet fédéral sur les litiges civils (Civil Litigation Research Project). De nombreux travaux ont concerné l'accès au droit, l'étude des jurys, le recours aux tribunaux. L'objectif était d'identifier les étapes franchies par les disputes, de leur formation jusqu'à leur formulation devant un tribunal. L'étude qui en sera tirée par trois sociologues, W. Felstiner, R. Abel, et A. Sarat deviendra une référence pour tous ceux qui s'attacheront ensuite à étudier les conditions du recours aux tribunaux. Cette enquête met en évidence trois moments dans la naissance d'un litige: l'identification du problème (naming), l'identification de la personne responsable (blaming), et la réclamation formée devant elle (claiming). Le litige proprement dit (avec le risque de saisine du tribunal), naît seulement après le rejet de la réclamation par la personne visée. L'étude a porté notamment sur le recours aux avocats et aux autres procédés de règlement disponibles dans la sphère sociale.

En Europe, c'est le sociologue allemand E. Blankenburg (1994a, b), qui a posé comme question centrale les conditions de la «mobilisation du droit». Les études se centrent sur les variables institutionnelles qui influent sur le comportement des demandeurs, abandonnant les explications strictement psychologiques. Ainsi, cherchant à expliquer pourquoi l'Allemagne présente des taux de recours beaucoup plus élevés que la Hollande, Blankenburg relève l'existence de nombreux procédés de filtrage dans le système hollandais, et conclut que l'infrastructure institutionnelle est suffisante pour expliquer pourquoi il est rationnel pour les allemands de recourir aux tribunaux, et pour les hollandais de les éviter (1994b).

101 Economie et Institutions $-\mathrm{n}^{\circ} 4-1^{\mathrm{e}}$ semestre 2004 
Cette sociologie apparaît soucieuse de vérifier la réalité de l'accessibilité des tribunaux, notamment à l'usage des plus démunis. Nulle critique de la "litigiosité », mais au contraire l'idée qu'une société saine est une société où les droits peuvent être défendus judiciairement, comme a pu l'écrire récemment un chercheur à propos de l'augmentation des contentieux au Japon (Seizelet, 2002).

La question de l'accès aux tribunaux reste donc au centre des questions de la sociologie de justice. Si les enquêtes empiriques se sont ralenties (du moins en Europe), on verra que le relais a été pris par les instruments juridiques, qui se sont fortement développés, notamment au plan communautaire.

\section{1-3 -2 La valorisation des alternatives au tribunal}

A la préoccupation de vérifier la réalité de l'accès aux tribunaux se substitue depuis une vingtaine d'années une approche opposée de valorisation des moyens de règlement des litiges ne faisant appel ni au droit ni aux tribunaux. Les sociologues et anthropologues du droit s'associent dans une même perspective de reconnaissance d'une justice non institutionnelle, délivrée par des tiers non professionnels. La médiation est créditée d'une valeur toute particulière, en tant que "moyen de développer un droit qui soit enraciné non dans l'action du législateur ou des magistrats, mais dans la société elle-même" (Bonafé-Schmitt, 1992). Dans le même sens, la vision dite "post moderne" du droit, développée par A.J Arnaud (1991), repose pour l'essentiel sur une analyse de la résistance sociale au droit étatique dont les manifestations sont recherchées dans les "justices informelles". Réformateurs et sociologues du droit aspirent au développement d'une justice immédiatement compréhensible par ceux à qui elle s'adresse, développant un véritable mythe de la justice communautaire. Ce courant se déclare hostile à l'intervention des professionnels dans les litiges, considérée comme la manifestation d'une "perspective hégémonique du juge". La préférence est donnée au tiers profane, censé être porteur des aspirations du groupe. Ce thème de «l'alternative » au droit et à la justice institutionnelle a trouvé un relais politique opportun avec les efforts de maîtrise des dépenses de justice. Au lieu d'être l'expression d'une défense des droits, l'action en justice est traitée comme une anomalie, produit d'une demande qui n'aurait pas trouvé son «médiateur naturel». Mais contrairement aux précédentes, ces approches sont pauvres en données empiriques systématisées, les juristes et sociologues intéressés développant davantage une théorie de ces recours que de leur pratique. Pour cette sociologie, le tribunal est un relais socialement inadéquat, assertion qui fait pendant à l'allégation d'inefficience portée par la Law and Economics. Il est 
donc inutile de développer une connaissance du procès, ce qui explique que ce courant, n'ait jamais produit de données empiriques sur la justice.

\section{1-3-3 Le tribunal placé au sein d'un système d'attentes économiques}

C'est dans la sociologie de Max Weber, attentive aux rapports entre le droit et l'économie, que la fonction économique du tribunal est posée de la manière la plus nette.

Cette fonction du tribunal est développée dans plusieurs points de l'œuvre de Weber, et notamment dans le premier chapitre d'Economie et société, consacré aux relations fondamentales entre l'économie et l'organisation sociale. Dans ce chapitre, Weber définit la règle de droit comme une prescription, assortie d'une garantie de coercition, consistant en «la présence d'une ou de plusieurs personnes dont la tâche est de faire prévaloir l'ordre juridique en utilisant les moyens de coercition spécialement prévus pour cela (contrainte juridique) » (Weber, 1995, T 2, pp. 13-14). Selon Weber, le développement du droit a conduit à une concentration des pouvoirs de contraintes entre les mains de l'Etat: "Actuellement, la contrainte juridique par la force est le monopole de l'Etat. Toutes les autres communautés qui exercent une contrainte juridique par la force sont considérées aujourd'hui comme hétéronomes (...)» (1995, T 2, p.15). Cette contrainte constitue la contrepartie du développement des droits subjectifs. Weber souligne que «l'économie moderne repose sur des chances acquises par contrats. (...), et que «l'intensité des échanges modernes exige un droit fonctionnant d'une manière prompte et sûre, c'est-à-dire garanti par la plus forte puissance de contrainte possible, et c'est ainsi que l'économie moderne, par son caractère propre, a contribué plus que toute autre chose à détruire les autres groupements sociaux qui étaient porteurs de droit et donc d'une garantie de droit. Tel a été le résultat du développement du marché » (1995, T 2, pp. 48-49). Du point de vue historique, l'évolution de ce pouvoir de commandement est allée dans le sens d'une concentration croissante au sein de l'appareil d'Etat, au détriment des autorités intermédiaires (corporations, communautés). Selon Max Weber, cette disparition progressive des foyers de production du droit est le moyen par lequel le droit peut servir efficacement l'économie : «la fusion de tous les groupements qui avaient été les agents d'une formation du droit dans la seule institution coercitive étatique se prétendant seule source de tout droit légitime montre de manière caractéristique la façon avec laquelle le droit sert les intérêts surtout économiques des parties en présence ». En ce sens, "un droit concret est envisagé comme "l'octroi d'un surcroît de chance pour que certaines attentes accordées à des individus pourvues de droits subjectifs par le droit objectif ne soient pas déçues " (1986, p.44). Les modalités pratiques de la mobilisation du droit sont examinées sous l'angle des maximes d'action.

103 Economie et Institutions $-\mathrm{n}^{\circ} 4-1^{\mathrm{e}}$ semestre 2004 
Pour Weber, les règles de droit sont des maximes pour différents acteurs intéressés à la règle, qu'il s'agisse des individus, ou des juges, l'ensemble constituant «l'être empirique du droit» (Weber, 2001, p. 148). L'action des juges qui se conforment à la maxime juridique est caractérisée par le fait qu'ils "se conforment à la maxime et décident des conflits d'intérêts en fonction d'une règle juridique déterminée ", celle des huissiers et policiers "qu'ils orientent leurs comportements sur la base de cette décision » (Weber, 2001, p. 149). C'est en appliquant ces maximes que les agents qui cherchent à modifier une situation empirique (par exemple, une cheminée qui fume), feront appel à un certain nombre de personnes (avocats, juges...), qui examineront sa demande d'un point de vue dogmatique, ce qui n'exclut pas que l'intéressé «perde devant le tribunal » (Weber, 2001, p. 150).

On retiendra cette dernière formule comme transition pour passer de la sociologie au droit. En effet, d'un point de vue économique, celui qui introduit une réclamation devant le tribunal peut échouer ou réussir. Mais en tant que sujet de droit, et quel que soit le résultat, il aura bénéficié de l'accès à un tribunal, dans des conditions qui sont aménagées tant par le droit interne que par le droit conventionnel.

\section{2- L'institution juridique de la garantie juridictionnelle des droits}

L'affirmation que le tribunal est un lieu de garantie des droits est un énoncé acceptable dans toutes les théories que nous venons de voir, même si l'efficience d'une sanction étatique est mise en doute, comme dans l'analyse économique du droit, ou si sa valeur sociale est contestée, comme dans la variante de la sociologie du droit qui se déclare hostile au procès. On peut voir dans cet accord minimal la reconnaissance des grands principes qui fondent la fonction de justice (2-1) et des règles qui en garantissent l'accès (22).

\section{2-1- L'obligation pour tout Etat de fournir un appareil de justice}

Dans la conception la plus modeste de la souveraineté étatique, l'Etat garantit les droits des citoyens en leur fournissant une police, une armée et une justice. L'article 12 de la Déclaration des droits de l'homme et du citoyen du 26 août 1789 en pose le principe : "La garantie des droits de l'Homme et du Citoyen nécessite une force publique: cette force est donc instituée pour l'avantage de tous, et non pour l'utilité particulière de ceux auxquels elle est 
confiée $» \cdot{ }^{6}$ La mise en mouvement de cette force est réservée à la puissance publique, qui peut l'exercer au soutien de sa propre action, ou à la demande des particuliers, par l'intermédiaire de l'appareil juridictionnel : d'une part en effet, les personnes morales de droit public, ou de droit privé chargées d'une mission de service public, peuvent se voir conférer, par l'autorité législative, le droit d'émettre des titres qui seront exécutoires en l'absence de recours ${ }^{7}$; d'autre part, les personnes privées, à qui est retiré le droit de se faire justice à elles-mêmes, bénéficient du "droit à un recours effectif », devant une autorité juridictionnelle indépendante, qui a le pouvoir d'ordonner l'exécution forcée de ses jugements.

Ces trois éléments, droit au recours, indépendance des juges, exécution forcée des jugements, constituent aujourd'hui les piliers de l'appareil de justice pour toute organisation démocratique.

Le droit à un recours effectif est reconnu par de multiples instruments juridiques à valeur constitutionnelle et internationale ${ }^{8}$. Dans le cadre de la Convention européenne des droits de l'homme, ce droit se décline en deux obligations principales qui pèsent sur les Etats: organiser un tribunal qui

6 Selon l'article 13 de cette même Déclaration, il s'agit là du premier motif du prélèvement de l'impôt : "Pour l'entretien de la force publique, et pour les dépenses d'administration, une contribution commune est indispensable : elle doit être également répartie entre tous les citoyens, en raison de leurs facultés".

7 Conseil constitutionnel, décision $\mathrm{n}^{\circ} 99-416$, DC du 23 juillet 1999, à propos d'une procédure d'exécution forcée pour le recouvrement de cotisations sociales: "si le législateur peut conférer un effet exécutoire à certains titres délivrés par des personnes morales de droit public, et le cas échéant, par des personnes morales de droit privé chargées d'une mission de service public, et permettre la mise en œuvre de mesures d'exécution forcée, il doit garantir au débiteur le droit à un recours effectif en ce qui concerne tant le bien-fondé desdits titres et l'obligation de payer que le déroulement de la procédure d'exécution forcée (...)".

8 Le droit au recours est garanti par l'article 16 de la Déclaration des droits de l'Homme et du citoyen ("Toute Société dans laquelle la garantie des Droits n'est pas assurée, ni la séparation des Pouvoirs déterminée, n'a point de Constitution "), par la Convention européenne des droits de l'homme et des libertés fondamentales, dans son article 13 ("Toute personne dont les droits et libertés reconnus dans la présente Convention ont été violés, a droit à l'octroi d'un recours effectif devant une instance nationale, alors même que la violation aurait été commise par des personnes agissant dans l'exercice de leurs fonctions officielles"), par la Cour de justice des communautés européennes, par la Charte des droits fondamentaux de l'Union européenne du 18 décembre 2001 dans son article 47 (3Toute personne dont les droits et libertés garantis par le droit de 1'Union ont été violés a droit à un recours effectif devant un tribunal (...)"), sans omettre les principes généraux du droit dégagés par le juge administratif, qui reconnaît depuis plus de cinquante ans un droit au recours (CE, 7 février 1947, D’Aillières, 79128, Rec. Lebon).

105 Economie et Institutions $-n^{\circ} 4-1^{\text {e }}$ semestre 2004 
puisse "trancher sur la base de normes de droit à l'issue d'une procédure organisée, toute question relevant de sa compétence ${ }^{9} »$; permettre un accès concret et effectif à un tribunal. Cette obligation peut nécessiter des mesures positives, « comme l'octroi d'une aide judiciaire gratuite, ou la simplification de la procédure dans les cas où il n'est pas possible à un particulier impécunieux de défendre utilement sa propre cause ${ }^{10}$. Le tribunal une fois saisi, le juge est tenu de juger, (article 4 du Code civil) ${ }^{11}$, et ce devoir de juger est garanti par $\mathrm{l}^{\prime} E \mathrm{Etat}^{12}$, «qui doit protection juridictionnelle à l'individu » (Favoreu, 1964, p. 37).

L'indépendance de l'autorité juridictionnelle, corollaire de la séparation des pouvoirs, s'énonce comme une interdiction faite au gouvernement et au législateur d'adresser des injonctions au juge. En ce qui concerne l'autorité judiciaire, l'indépendance est garantie par le titre VIII de la Constitution du 4 octobre 1958, et plus spécialement par son article $64^{13}$. Pour la juridiction administrative (qui comprend les tribunaux et cours administratives ordinaires ou spécialisées), le principe d'indépendance, moins naturel en raison de la proximité de ces juridictions avec l'Etat, est rangée par le Conseil constitutionnel dans la liste des "principes fondamentaux reconnus par les lois de la République $» .^{14}$

L'efficacité du recours, garanti par les mêmes instruments internationaux, s'entend comme l'existence d'une contrainte pour l'exécution des décisions. La Cour européenne des droits de l'homme définit le tribunal comme l'organe "habilité à rendre une décision contraignante sur la cause dont il est saisi ${ }^{15} »$. Les juges sont ainsi détenteurs du pouvoir de rendre efficaces leurs jugements en en ordonnant l'exécution. Ce lien entre le jugement et la force publique est lisible dans la formule qui confère, en

9 Bellilos c/ Suisse du 29 avril $1988 \S 64$.

10 Airey c/ Irlande du 9 octobre 1979 § 24-25.

11 Article 4 du code civil : "Le juge qui refusera de juger, sous prétexte du silence, de l'obscurité ou de l'insuffisance de la loi, pourra être poursuivi comme coupable de déni de justice. "

12 L'Etat est tenu de réparer le dommage causé par le fonctionnement défectueux de la justice (article L. 781-1 du Code de l'organisation judiciaire).

13 "Le Président de la République est garant de l'indépendance de l'autorité judiciaire. Il est assisté par le Conseil Supérieur de la Magistrature. Une loi organique porte statut des magistrats. Les magistrats du siège sont inamovibles".

14 Conseil constitutionnel, décision $\mathrm{n}^{\circ} 80-119$, DC du 22 juillet 1980, concernant une loi portant validation d'actes administratifs et qui modifiait la législation applicable à des instances pendantes devant des juridictions administratives : "Il n'appartient ni au législateur ni au Gouvernement de censurer les décisions des juridictions, d'adresser à celles-ci des injonctions et de se substituer à elles dans le jugement des litiges relevant de leur compétence"

15 Sramek c/ Autriche du 22 octobre $1984 \S 36$.

Economie et Institutions $-n^{\circ} 4-1^{e}$ semestre 2004 
France, force exécutoire aux jugements ayant acquis force de chose jugée : il s'agit d'un commandement adressé à l'autorité publique de prêter mainforte à leur exécution ${ }^{16}$.

Ainsi, de la création des tribunaux, à l'aménagement de leur accès jusqu'à la mise en œuvre de la force publique pour l'exécution de leurs décisions, l'évidence est que l'Etat est le support de l'efficacité de la garantie des droits promise par la Déclaration des droits de l'Homme. On peut exprimer cette idée en termes économiques, en disant que dans une économie de marché fondée sur la détention de droits, les tribunaux sont des opérateurs de transformation de prétentions juridiques en titres exécutoires, et que c'est aux Etats de fournir les moyens juridiques et économiques propres à assurer le fonctionnement efficace de cet appareil.

\section{2-2 L'aménagement des droits d'action}

Mais quels droits peuvent se voir reconnaître une garantie? L'idée la plus répandue est que l'Etat prêterait son appareil coercitif pour " punir » quiconque "violerait» un droit dont un sujet serait titulaire. Les réalistes scandinaves ont critiqué dans cet énoncé la notion de "violation du droit » en tant qu'elle signifierait une désobéissance à un commandement : si c'était le cas, la sanction infligée par le tribunal devrait être prononcée au profit de la collectivité, et non du sujet de droit. Mais on peut critiquer aussi la deuxième partie de l'énoncé : la garantie étatique ne doit-elle être fournie que si les droits ont été certainement méconnus? L'admettre reviendrait à n'accueillir devant les tribunaux que les porteurs de droits incontestés, et à faire du tribunal un simple distributeur de sanctions. L'examen du droit processuel invalide ce schéma simplifié. On y voit un droit d'action émancipé des droits substantiels, dont l'extension est aménagée par des règles spécifiques.

16 Cette formule est actuellement issue du décret $n^{\circ} 47-1047$ du 12 juin 1947 : "En conséquence, la République française mande et ordonne à tous huissiers de justice pour ce requis de mettre ledit arrêt (ou ledit jugement, etc.) à exécution, aux procureurs généraux et aux procureurs de la République près les tribunaux de grande instance d'y tenir la main, à tous commandants et officiers de la force publique de prêter main-forte lorsqu'ils en seront également requis". Elle est reprise pour les juridictions administratives par l'article R751-1 du code des tribunaux administratifs : " Les expéditions de la décision délivrées aux parties portent la formule exécutoire suivante : "la République mande et ordonne au (indiquer soit le ou les ministres, soit le ou les préfets désignés par la décision) en ce qui le (les) concerne ou à tous huissiers de justice à ce requis en ce qui concerne les voies de droit commun contre les parties privées, de pourvoir à l'exécution de la présente décision ".

107 Economie et Institutions $-\mathrm{n}^{\circ} 4-1^{\mathrm{e}}$ semestre 2004 


\section{2-1-1 Définitions du droit d'action}

En droit interne, ce que l'on dénomme dans le langage de la procédure droit d'action est défini par l'actuel article 30 du nouveau code de procédure civile : «l'action est le droit, pour l'auteur d'une prétention, d'être entendu sur le fond de celle ci afin que le juge la dise bien ou mal fondée. Pour l'adversaire l'action est le droit de discuter le bien-fondé de cette prétention». Ce droit est un droit subjectif distinct du droit réclamé, et consiste essentiellement à être admis à soutenir une prétention devant un juge, en demande ou en défense. Si on observe le contenu de ce droit subjectif processuel, on constate qu'il assure aux porteurs de simples intérêts les mêmes garanties que celles qui sont assurées aux détenteurs de droits classiques. Les uns comme les autres bénéficient du droit d'être entendu sur le fond de leurs prétentions, lesquelles ne sont rien d'autre que le nom donné à la réparation des intérêts lésés. Cette aptitude à regrouper droits et intérêts est la seule justification pratique du droit subjectif d'action, dont on a pu dire par ailleurs qu'il crée d'inutiles complications dans le régime des défenses, exceptions et fins de non-recevoir ${ }^{17}$.

Le droit d'action est également reconnu de manière autonome dans la Convention européenne des droits de l'homme. L'article $6 \S 1$ de cette convention pose que «toute personne $a$ droit à ce que sa cause soit entendue équitablement, publiquement et dans un délai raisonnable, par un tribunal indépendant et impartial, établi par la loi, qui décidera, soit des contestations sur ses droits et obligations de caractère civil, soit du bien-fondé de toute accusation en matière pénale dirigée contre elle (...)». Ce droit d'être entendu est indépendant de l'existence d'un droit expressément reconnu en droit interne. Pour la Cour européenne, il suffit qu'un droit soit défendable en droit interne pour entraîner l'application de l'article $6 \$ 1$. Ce critère permet à la Cour de s'affranchir de la volonté des Etats de ne reconnaître l'application de la Convention que si leur droit interne leur alloue un droit spécifique ${ }^{18}$. A partir du droit d'action largement conçu, les

17 Selon Raymond Martin, "le droit d'action a crée une complexité inutile dans le régime des demandes: Plutôt que de parler de droit d'action, il vaudrait mieux s'en tenir à la notion de demande en justice, et considérer que les critères de qualité et d'intérêt par exemple sont des conditions de recevabilité des demandes ".

18 James c/ Royaume-Uni, 21 février 1986, § 80. Dans cet arrêt, était en cause la législation britannique sur les conditions du rachat d'un logement par des preneurs d'un bail. La loi ne laissait aucun moyen au propriétaire, une fois réunis les critères définis, pour contester le droit des preneurs au rachat. L'absence de possibilité de saisir valablement un tribunal avait été considérée comme constitutive d'une violation de l'article $6 \S 1$.

Economie et Institutions $-n^{\circ} 4-1^{\text {e }}$ semestre 2004 
intérêts les plus diversifiés vont revendiquer "l'écoute" d'un tribunal, ouvrant à une casuistique çà et là ponctuée par des interventions étatiques.

\section{2-1-2 Extension du droit d'action}

Le droit d'action n'est pas inconditionnel. L'article 31 du nouveau code de procédure civile le confère «à tous ceux qui ont un intérêt légitime au succès ou au rejet d'une prétention, sous réserve des cas dans lesquels la loi attribue le droit d'agir aux seules personnes qu'elle qualifie pour élever ou combattre une prétention, ou pour défendre un intérêt déterminé» ${ }^{19}$. Si la condition de l'intérêt est générale, celle de la «qualification» dépend de l'existence d'une loi spéciale qui réserve l'action à certaines parties. Du point de vue procédural, la contestation du droit d'action est ouverte au défendeur à l'action, sous forme d'une fin de non recevoir, allouée par l'article 122 du même code : "Constitue une fin de non-recevoir tout moyen qui tend à faire déclarer l'adversaire irrecevable en sa demande, sans examen au fond, pour défaut du droit d'agir, tel le défaut de qualité, le défaut d'intérêt, la prescription, le délai préfix, la chose jugée". Si la distinction des fins de non recevoir, des exceptions et des défenses au fond n'est pas toujours cohérente, 20 il importe de voir que l'existence d'un droit d'action autonome tient ouvert en permanence ${ }^{21}$ un débat sur la légitimité de la prétention des parties. Selon le type de litiges, ce débat portera sur l'une des deux causes qui fondent le droit d'action : la détention d'un droit d'action spécifique, un intérêt pour agir.

\section{Des droits d'action attitrés}

Attribuer par un texte un droit d'action à des personnes déterminées est un moyen efficace d'assurer leur présence sur la scène judiciaires. Dans certains cas, les actions sont réservées à des personnes déterminées, à

19 On remarquera que cette formule est très proche de la définition que donnait Jhering du droit subjectif lui-même, comme "intérêt légitime juridiquement protégé" (1880, p. 326-327). Pour ce romaniste, le droit est un instrument de protection des intérêts, et le tribunal, le lieu par excellence où les droits se réalisent. L'action en justice était pour lui non seulement un droit, mais une obligation, car "transiger sur un droit bafoué afin de s'épargner les frais d'un procès, c'est déserter devant un combat nécessaire ".

20 Notamment entre les causes de nullité pour irrégularités de fond (art. 117), et les fins de non recevoir (art. 122). Pour une critique de ces classifications, v. R. Martin préc., p. 420.

21 Les fins de non-recevoir peuvent être présentées à toute hauteur de la procédure (art. 123), règle qui peut surprendre s'agissant de faire obstacle à l'examen du fond du droit, mais qui s'explique par l'histoire de cette catégorie qui n'était initialement qu'une variante des défenses au fond.

109 Economie et Institutions $-n^{\circ} 4-1^{e}$ semestre 2004 
l'exclusion de toutes autres. Ce que l'on appelle les "actions attitrées" se rencontrent notamment dans le droit de la filiation et du mariage (époux dans l'action en divorce, enfant dans la recherche de paternité naturelle, personne protégée dans l'action en nullité relative, etc.) On s'intéressera davantage aux situations où la loi, en attitrant certaines personnes, introduit volontairement de nouveaux acteurs dans le cadre du procès, aux côtés des acteurs traditionnels, souvent pour en renforcer l'action. Le domaine d'élection de ce procédé est celui des groupements : associations, syndicats, instances représentatives du personnel.

Hors les cas où leurs intérêts personnels sont en jeu, les associations peuvent se voir reconnaître par la loi un intérêt à agir pour défendre l'intérêt général. Les articles 2-1 et s. du Code de procédure pénale accordent ainsi les droits reconnus à la partie civile à une série d'associations définies par leur objet, et pour des infractions déterminées (infractions contre le racisme, la délinquance routière, la toxicomanie, les violences sexuelles....). L'article L. 252-3 du code rural reconnaît les mêmes droits aux associations agréées de protection de la nature et de l'environnement. En matière de consommation, la mise en place d'acteurs collectifs est devenue une obligation communautaire, notamment avec la directive 98/27/CE du 19 mai 1998 « relative aux actions en cessation en matière de protection des intérêts des consommateurs». Des «entités qualifiées » se voient habilitées aux fins d'exercer des actions en cessation au nom de l'intérêt collectif des consommateurs. Transposant cette directive, l'ordonnance 2001-741 du 23 août 2001 a introduit un article dans le code de la consommation (art. L.4216), qui donne aux associations agréées et à certains organismes inscrits sur une liste spéciale, le pouvoir d'agir devant les juridictions civiles « pour faire cesser ou interdire tous agissements illicites au regard des dispositions transposant la directive 98/27/CE », en l'absence d'action exercée par un consommateur. Le juge peut à ce titre ordonner la suppression d'une clause illicite ou abusive dans tout contrat ou type de contrats proposé ou destiné au consommateur, mécanisme qui vise à pallier tant l'insuffisance que la dispersion des actions individuelles des consommateurs. On aurait garde de négliger l'incidence de ces multiples qualifications de plaideurs. En allouant des droits d'actions à ces associations, la loi les transforme en agents actifs des politiques concernées, sous quelque forme que ces pouvoirs soient exercés (recours effectif ou simple menace).

Le droit d'action des syndicats est libéralement admis depuis un arrêt des chambres réunies du 5 avril 1913 leur reconnaissant le pouvoir "d'exercer tous les droits réservés à la partie civile, relativement aux faits portant un préjudice direct ou indirect à l'intérêt collectif de la profession qu'ils représentent» (aujourd'hui, l'article art. L. 411-11 code trav.). A ce droit d'action général pour la défense des intérêts de la profession, la loi en a ajouté d'autres, limités à certains contentieux. L'action dite en

Economie et Institutions $-n^{\circ} 4-1^{e}$ semestre 2004 
«substitution», autorise le syndicat à exercer l'action individuelle d'un salarié, sans avoir à justifier d'un mandat, mais à condition que ce dernier ne s'y soit pas opposé. Il n'existe pas de principe général de recours, mais une multitude de textes spécifiques dans le code du travail ${ }^{22}$. Cette action en substitution constitue un levier puissant de contrôle de l'application des règles du code du travail dans l'entreprise, parfois contre l'intérêt propre du salarié. De plus, l'article L. 135-5 donne aux organisations et groupements liés par une convention ou un accord collectif de travail le pouvoir "d'intenter toute action visant à obtenir l'exécution des engagements et le cas échéant, des dommages -intérêts ».

Le syndicat n'est pas le seul à se voir reconnaître un pouvoir d'action au sein de l'entreprise. Les délégués du personnel peuvent ainsi recourir à une procédure d'alerte pour assurer dans l'entreprise la protection des droits des personnes (art. L. 422-1-1 du code du travail).

\section{Un intérêt pour agir}

En-dehors des actions attitrées, toute prétention, pour être recevable, doit être fondée sur un intérêt. En droit interne comme en droit conventionnel, le débat sur l'intérêt recevable est une voie de contrôle de l'arrivée de nouveaux plaideurs devant les tribunaux.L'intérêt pour agir en droit interne

La notion d'intérêt se définit par rapport à l'utilité que la procédure peut présenter pour le plaideur, que l'avantage soit matériel ou moral. Sur une base aussi large, les intérêts qui cherchent à se faire reconnaître devant les tribunaux sont en nombre infini. La doctrine juridique qui s'efforce de synthétiser les solutions jurisprudentielles retient plusieurs critères de l'intérêt recevable : il doit être légitime, né et actuel, direct, personnel. On puisera dans l'abondante jurisprudence interne pour illustrer quelques une des causes d'irrecevabilité.

En requérant un « intérêt légitime », l'article 31 du nouveau code de procédure civile a conservé la trace du fondement subjectiviste de l'action : dire d'un intérêt qu'il doit être légitime pour que l'action soit recevable pourrait être compris comme l'exigence que l'action soit fondée sur un droit, alors que le but de l'action est précisément d'obtenir que ce droit soit reconnu par le juge. La confusion a bien été perçue en doctrine comme en jurisprudence, et la portée du critère de légitimité est aujourd'hui très réduite. L'exemple le plus fameux d'une discussion sur la légitimité reste celui de la jurisprudence dite « de la concubine». La Cour de cassation avait

22 Le code du travail cite notamment : le statut de travailleur à domicile (art. 721-19), l'application d'une convention collective (L. 135-4), le travail temporaire (L. 124-20), le CDD (L. 122-3-16) l'égalité professionnelle homme femme (L. 123-6) ou les droits des travailleurs étrangers (L. 341-6-2)

111 Economie et Institutions $-n^{\circ} 4-1^{e}$ semestre 2004 
longtemps déclaré irrecevable l'action de la concubine en réparation du préjudice causé par la mort de son compagnon, au motif de l'absence de lien de droit entre eux. On voit sans peine le glissement de sens qui a pu s'établir entre le caractère "illégitime» du concubinage (aux yeux de nombre de juristes de l'époque), et l'illégitimité de l'action. Mais il s'agissait là d'une pure appréciation morale, et le lien entre le concubinage et l'intérêt illégitime a été rompu par un arrêt de la Chambre Mixte du 27 février 1970, énonçant dans un attendu célèbre « que l'article 1382 du code civil, en ordonnant que l'auteur de tout fait ayant causé un dommage à autrui sera tenu de le réparer, n'exige pas, en cas de décès, l'existence d'un lien de droit entre le défunt et le demandeur $»^{23}$.

Hors les causes d'irrecevabilité de l'action, le critère de légitimité de l'intérêt trouve un autre terrain d'application avec les condamnations à dommages intérêts du demandeur en cas de procédure abusive: le but exclusivement dilatoire de l'action transforme en abus le droit d'agir en justice ${ }^{24}$.

La condition d'un intérêt né et actuel condamne les actions fondées sur un préjudice simplement éventuel, qu'elles soient préventives, ou déclaratoires, c'est-à-dire tendant à obtenir du juge qu'il déclare une situation juridique. Par exception, de multiples textes autorisent des actions qui encourraient le reproche d'éventualité : actions conservatoires, actions en déclarations de nationalité, référés préventifs...Mais à l'inverse de la condition de légitimité, la condition d'actualité est efficacement utilisée par les défendeurs, notamment en matière sociale. Il a été ainsi jugé par la Chambre sociale de la Cour de cassation que des salariés qui font partie d'un projet de licenciement collectif pour motif économique n'étaient pas recevables à agir en référé pour demander la nullité du plan ${ }^{25}$.

L'intérêt personnel ne se confond pas avec l'intérêt individuel, et les associations peuvent agir à l'égal des particuliers, qu'elles défendent leurs droits en tant que groupements ou comme collection d'intérêts individuels. C'est sur cette base «individualiste» qu'est admis l'intérêt pour agir d'associations créées en vue de défendre l'intérêt de leurs membres. L'assimilation entre les personnes morales et les personnes physiques est alors complète du point de vue de l'action.

23 Ch. Mixte, 27 février 1970, Bulletin des arrêts Cour de Cassation, $n^{\circ} 1$ p.1;

Dalloz, les grands arrêts de la jurisprudence civile, observations H. Capitant, A. Weill, F.Terré, p. 419, Dalloz 1970 p. 201, note Combaldieu, Revue Générale des assurances terrestres 1970 p. 195, note A.B, Gazette du Palais 1970 p. 163 , note $X$.

24 Civ. 2ème, 6 mars 2003, pourvoi n01-02-745, Bull. civ, Bull. d'information, pour un cas de demande de remboursement de frais d'exécution forcée à l'encontre d'un huissier.

25 Cass. Soc., 15 janvier 2003, Lamy, Bull. civ.

Economie et Institutions $-n^{\circ} 4-1^{e}$ semestre 2004 


\section{Les intérêts recevables en droit conventionnel et communautaire}

De manière générale, le droit conventionnel exige que les droits des Etats ouvrent largement le droit d'action, craignant qu'une conception trop étroite de la recevabilité mette en péril l'accès effectif au tribunal. C'est ce motif qui a inspiré la Cour européenne des droits de l'homme dans sa condamnation de la France en 1995 dans une affaire d'indemnisation d'un hémophile contaminé par le VIH à la suite d'une transfusion. La Cour de Cassation avait jugé que la victime, déjà indemnisée par un fonds d'indemnisation, n'avait plus intérêt pour agir devant les juridictions judiciaires pour demander un complément d'indemnisation, et avait confirmé l'irrecevabilité de la demande. Ce refus constituait, selon la Cour européenne, une méconnaissance du «droit d'accès concret et effectif à un tribunal» ${ }^{26}$. Il est évident que c'est l'emploi même de la formule "défaut d'intérêt pour agir» qui a suscité une réaction négative de la Cour européenne, qui l'a entendue comme un refus d'examen de la demande, proche du refus du droit d'accès au tribunal. Le glissement opéré par la Cour européenne met l'accent sur l'importance du jugement porté sur le fondement d'un intérêt, jugement qui implique un examen complet de la cause par un tribunal, même si le résultat final est négatif pour le demandeur.

Devant les instances communautaires, le contrôle de l'action est plus strict. L'article 173 du Traité subordonne la recevabilité d'un recours à la preuve d'une atteinte directe et individuelle, ainsi que d'un intérêt pour agir. Un arrêt du Tribunal de première instance des communautés européennes du 27 avril 1995 rendu à propos de la recevabilité de l'action d'un syndicat en matière de concentration a considéré qu'un tel intérêt n'existait pas au regard de la finalité du règlement relatif aux concentrations, lequel "tend à préserver et à développer une concurrence effective sur le marché commun"27. Dans cet arrêt, statuant sur la recevabilité d'un recours formé par un syndicat contre une décision de la Commission concluant à la compatibilité d'une opération de concentration avec le marché commun, il a été jugé que la décision contestée ne concernait les représentants des travailleurs des entreprises en cause que dans une mesure très limitée: ces représentants figurent certes expressément parmi les tiers justifiant d'un intérêt suffisant pour être entendus par la Commission (au sens du règlement 4064/89), mais le droit de recours des représentants est limité à la défense de ces seuls intérêts procéduraux, la qualité pour agir se limitant à

26 CEDH, arrêt Bellet c/France, 4 déc. 1995, J.CP. 1996.3910, n² 21, obs. Sudre. Confirmé depuis par F.E c/ France, 30 oct. 1998, Clunet 1999, 235, obs. P. Tavernier.

27 TPI, 27 avril 1995, T-96/92, Comité central d'entreprise de la Société générale des grandes sources contre Commission des Communautés européennes.

113 Economie et Institutions $-\mathrm{n}^{\circ} 4-1^{\mathrm{e}}$ semestre 2004 
faire examiner par le juge communautaire si les garanties procédurales assurées pour le cours de la procédure ont été ou non méconnues . "Les intérêts légitimes des tiers qualifiés, c'est-à-dire de ceux qui sont uniquement susceptibles de subir les effets incidents de la décision de la commission sur leur sphère juridique, n'exigent pas qu'ils bénéficient de garanties identiques à celles qui sont accordées aux personnes intéressées par l'opération de concentration en vue d'assurer le respect des droits de leurs droits de la défense dans le déroulement de ladite procédure". Le Tribunal a donc dénié au syndicat tout intérêt légitime à agir au regard des objectifs fondamentaux du traité, et donc à voir ses demandes prises en considération par la Commission.

Un arrêt de la Cour de justice des communautés européennes du 2 avril $1998^{28}$ a de même déclaré irrecevable le recours de l'Association Greenpeace tendant à l'annulation d'une décision de la Commission de verser à l'Espagne une somme au titre du concours financier apporté par le Fonds européen de développement régional à la construction de deux centrales électriques aux Canaries. Ce recours avait été formé en application d'une directive. C'est en raison du caractère indirect du lien entre la décision de la commission et la construction des centrales que ce droit de recours a été refusé, selon une jurisprudence constante de cette Cour. La CJCE renvoie les intéressés à agir "devant leurs juridictions nationales pour contester les décisions administratives relatives à la construction des centrales".

On retiendra de ces quelques exemples que la détermination des personnes qui peuvent faire entendre leurs prétentions devant un tribunal est un procédé clé du contrôle des situations économiques, qui s'étend au territoire de l'Union européenne. Notamment, la qualification des personnes admises à agir constitue une pièce essentielle du dispositif de mise en œuvre de certaines catégories de droits.

\section{3- Le recours aux tribunaux comme action économique}

Nous l'avons relevé, la garantie procédurale des droits est d'abord assurée en droit interne par l'aménagement d'un droit d'accès effectif au tribunal, et ce droit constitue aujourd'hui une liberté publique garantie par les instruments internationaux. Le sujet passif de ce droit est toujours une entité, Etat ou collectivité. Mais le marché se pense plus que jamais dans le cadre élargi de la Communauté européenne. Le phénomène le plus intéressant dans ce cadre est celui du développement d'un droit procédural européen voué à assurer la garantie de droits et intérêts acquis en n'importe

28 CJCE, Stichting Greenpeace Council contre Commission des communautés européennes, 2 avril 1998, aff. C-321/95 P.

Economie et Institutions $-n^{\circ} 4-1^{e}$ semestre 2004 
quel point du territoire de la Communauté. Le chemin parcouru sur ce point entre le Traité de Rome et le Traité d'Amsterdam adopté le 2 octobre 1997, est considérable. La Communauté initialement conçue avait reçu mission de mettre en œuvre une politique de libre circulation des marchandises, des personnes, des capitaux et des travailleurs, et ne comportait des dispositions sur la justice que dans des domaines particuliers, comme la coopération judiciaire civile, la consommation, et l'environnement, laissant aux Etats membres le soin d'assurer la mise en œuvre judiciaire des droits. Le Traité d'Amsterdam complétait ces fonctions en donnant à l'Union la mission de maintenir et de développer un espace de liberté de sécurité et de justice, en exigeant «non plus une coopération entre Etats, mais une véritable intégration » (Rouchaud-Joet, 2003). Mieux, la coopération judiciaire civile est sortie de ce qu'on appelait le «troisième pilier » et du Traité sur l'Union européenne, pour entrer dans le Traité instituant la Communauté européenne, avec le développement corrélatif d'instruments contraignants comme le règlement et la directive, la consultation obligatoire du Parlement, et le rôle interprétatif de la Cour de justice des communautés européennes. L'article $61 \mathrm{du}$ Traité CE donnait au Conseil un délai de 5 ans pour prendre des mesures pour la mise en place de cet « espace de liberté, de sécurité et de justice », notamment dans le domaine de la coopération judiciaire civile, visée à l'article 65 du même Traité.

Le programme de travail ainsi ouvert donnait lieu à un train impressionnant de dispositions, toutes orientées vers l'accroissement de l'efficacité de l'appareil de justice des Etats. Le Conseil européen de Tampere de 1999 a énuméré ces mesures, dans le but clairement exprimé de «faire en sorte que l'incompatibilité ou la complexité des systèmes juridiques et administratifs des Etats membres n'empêche ou ne dissuade pas les particuliers et les entreprises d'exercer leurs droits ». Autrement dit, pas de marché sans droits et sans garantie des droits.

Avec le Traité d'Amsterdam, les bases juridiques offertes par le Traité CE pour intervenir en matière de procédure se sont diversifiées. Aujourd'hui, la Commission et le Conseil peuvent proposer des mesures sur ce point tant au titre de la consommation, de l'environnement, de l'achèvement du marché intérieur, que de la coopération judiciaire civile.

\section{3-1 Un appareil juridictionnel incitatif pour les consommateurs}

\section{3-1-1 L'intérêt du marché pour les recours des consommateurs à la justice}

La reconnaissance d'une position d'infériorité des consommateurs dans la relation économique a pour corrélat l'attribution de droits qui leur sont spécifiques, visant à contrebalancer le poids économique des opérateurs, notamment à la phase de conclusion des contrats. Or de tels droits ne sont économiquement efficaces que si leur mise en œuvre est

115 Economie et Institutions $-n^{\circ} 4-1^{\text {e }}$ semestre 2004 
effective. Ce point avait été souligné par la Commission dans son premier Livre vert du 11 novembre 1993 : « à tout droit doit correspondre une action destinée à le faire valoir en justice, en contrepartie de l'interdiction de faire justice à soi même ${ }^{29}$. En agissant en justice, le consommateur ne se limite pas à défendre ses droits : il assure une véritable fonction, contribuant à la régulation du marché : on ne s'étonnera pas que l'article 153, point 3, lettre a, du Traité, place en première position pour assurer les objectifs élevés de protection des consommateurs, les «mesures que [la communauté] adopte en application de l'article 95 dans le cadre de la réalisation du marché intérieur».

Reste que les consommateurs individuels ne sont pas toujours des agents actifs du contrôle des activités des professionnels. Pour la Commission, l'explication de l'inertie des consommateurs est financière: le non exercice des poursuites serait l'effet de la « disproportion entre l'enjeu économique de l'affaire et le coût de son règlement judiciaire ${ }^{30}$. Pallier cette inertie par l'intervention communautaire n'était alors guère aisé, en raison notamment du fait que les coûts de procédure dépendent de l'état des droits internes, qui n'étaient pas directement accessibles à l'action communautaire. Avec le Traité d'Amsterdam, l'accès des consommateurs à la justice et au droit vient de trouver un nouveau support juridique. Ainsi la directive 2003/8/CE du Conseil du 27 janvier 2003 vise à «améliorer l'accès à la justice dans les affaires transfrontalières par l'établissement de règles minimales communes relatives à l'aide judiciaire accordée dans le cadre de telles affaires ». ${ }^{31}$ Prise sur le fondement des articles 61 point c et $67 \mathrm{du}$ Traité $\mathrm{CE}$, concernant la coopération judiciaire dans les matières civiles ayant une incidence transfrontière, ainsi que sur l'article $65 \mathrm{c}$ du Traité CE, qui vise à «éliminer les obstacles au bon déroulement des procédures civiles, au besoin en favorisant la compatibilité des règles de procédure civile applicables dans les États membres ", cette directive pose des normes minimales communes dans le domaine des litiges transfrontaliers, en matière civile et commerciale. Son objectif est clairement de pallier les effets désincitatifs du coût de la procédure sur l'action, et s'adresse à la partie la plus démunie. Dans le considérant 6, il est affirmé que "Le manque de ressources d'une personne partie à un litige, qu'elle soit demanderesse ou défenderesse, pas plus que les difficultés induites par le caractère transfrontalier d'un litige, ne devraient constituer des obstacles à un accès effectif à la justice ». Par ailleurs, il est attendu des Etats qu'ils prévoient une

29 Point III.D.1 du Livre Vert du16 novembre 1993 sur l'accès des consommateurs à la justice et le règlement des litiges de consommation dans le marché unique (COM(93)576 final).

30 Cette expression figure de nombreux actes communautaires concernant les droits des consommateurs depuis une dizaine d'années.

31 JOCE, ${ }^{\circ} \mathrm{L}$ 026, 31/01/2003, pp. 41-47.

Economie et Institutions $-n^{\circ} 4-1^{e}$ semestre 2004 
aide judiciaire élargie à l'amont du procès, couvrant «les conseils précontentieux afin de parvenir à un règlement avant d'engager une procédure judiciaire (...)» (considérant 11). Cette extension entre dans la logique du droit communautaire de la consommation, qui tient compte de l'aversion au risque de ces derniers en leur proposant des mesures amiables palliatives.

\section{3-1-2 Les règlements amiables comme palliatifs}

Parallèlement au développement $\mathrm{d}$ 'instruments visant à abaisser le coût d'entrée dans une procédure judiciaire, les procédures amiables ont pris place au rang des moyens complémentaire d'accès au droit. La démarche a son origine dans le droit de la consommation, et s'est développée essentiellement dans ce secteur. Dès 1975, la Commission organisait un colloque sur les "moyens judiciaires et parajudiciaires de la protection des consommateurs ", d'où résultait une liste de cinq procédés : " renforcement de la formation et de l'information du consommateur, instauration d'organismes de conciliation, création d'instances d'arbitrage, simplification des procédures judiciaires pour les litiges portant sur de petites sommes, prises en charge de la défense des consommateurs par des centrales de consommateurs ou des services publics de type ombudsman $»^{32}$. Depuis lors, l'expression «accès à la justice du consommateur » s'entend de manière extensive "comme accès pratique à ses droits, et non l'accès à la justice stricto sensu, à savoir, aux tribunaux »33. En 1993, a été édité un Livre Vert sur l'accès des consommateurs à la justice et le règlement des litiges de consommation dans le marché unique (Com(93) 576 du16 nov. 1993), qui mettait l'accent sur les procédés de résolution extrajudiciaires compte tenu $\mathrm{du}$ coût $\mathrm{d}$ 'accès aux tribunaux. A la suite de ce Livre vert, la Commission a émis une Recommandation (98/257CE), «concernant les principes applicables aux organes responsables pour la résolution extra- judiciaire des litiges de consommation ${ }^{34}$. L'incitation à recourir aux modes de résolution extra- judiciaires se fondait notamment sur le considérant que «la majorité des litiges de consommation, de par leur nature, se caractérisent par une disproportion entre l'enjeu économique de l'affaire et le coût de leur règlement judiciaire ». L'idée de « faible valeur » est à comprendre dans une

32 Les conclusions de ce colloque ont été rappelées dans la résolution du Conseil du 19 mai 1981 relative au programme de politique de protection et d'information des consommateurs ( $\mathrm{JO} \mathrm{n}^{\circ} \mathrm{C} 133$ du 03/06/1981, p. 1 et s., $\left.\mathrm{n}^{\circ} 38\right)$

33 Voir le rappel de ce principe dans la communication COM (1998)198.

34 Faisant suite elle-même au Livre Vert sur l'accès des consommateurs à la justice et le règlement des litiges de consommation dans le marché unique (Com(93) 576 du16 nov. 1993).

117 Economie et Institutions $-n^{\circ} 4-1^{e}$ semestre 2004 
balance coût/bénéfice, plus qu'à une appréciation de la valeur intrinsèque de la créance.

Loin de vouloir détourner le consommateur de toute revendication juridique, les procédures amiables constituent une incitation à réagir adressée à un consommateur considéré comme "averse» au risque judiciaire. Dans ce rapport déséquilibré, il s'agit de donner à la partie faible les moyens de constituer une force qui puisse compter sur un marché concurrentiel.

\section{3-2 Un appareil juridictionnel efficace pour tous les opérateurs du marché}

Si les consommateurs sont incités à exercer activement leurs droits, en saisissant les tribunaux, ou en formulant des plaintes auprès d'entités qualifiées, les professionnels sont également incités à user de leurs droits dans le cadre du marché. On pense bien sûr au droit de la concurrence, et à son cortège $\mathrm{d}$ 'autorités et de juridictions spécialisées, auxquelles sont confiés des pouvoirs étendus pour corriger les distorsions de marché ${ }^{35}$. Mais au-delà de ce droit spécial, le marché requiert la mobilisation d'instruments de droit commun destinés à fournir aux détenteurs de créances impayées, usagers des tribunaux, l'obtention rapide et efficace de titres exécutoires. Divers instruments ont été mis au point en droit communautaire, qui prennent appui, selon les procédures envisagées, sur le marché intérieur ou la coopération judiciaire.

\section{3-2-1 Des procédures efficaces dans le cadre d'un marché intérieur}

Différents instruments ont été élaborés au cours de la période récente, dans le droit fil des conclusions d'un groupe de travail qui avait abordé les règles de procédures sous l'angle de la mise en place d'un marché intérieur (Storme, 1995). Les initiatives ont en effet été prises sur le fondement de l'article $95 \mathrm{du}$ Traité CE. C'est le cas de la directive du 29 juin 2000 concernant la lutte contre le retard de paiement dans les transactions commerciales ${ }^{36}$. Dans son considérant 16 , cette directive relevait que les

35 Pour prendre un exemple récent, le rapport du groupe d'experts chargé de traiter des relations entre producteurs et distributeurs a prôné le renforcement du dispositif administratif et judiciaire de mise en œuvre de la réglementation. Il est notamment prévu l'incitation du recours au juge civil et au juge pénal, mais sur un base rénovée, ces juges devant être formés en vue de les adapter au traitement de ce contentieux spécifique. Rapport d'expertise sur les relations entre industrie et commerce sous la direction de Guy Canivet, premier président de la Cour de cassation, remis 18 octobre 2004 au Ministre des Finances.

36 Directive 2000 /35/CE, JO L 200 du 08-08-2000, p. 35.

Economie et Institutions $-n^{\circ} 4-1^{e}$ semestre 2004 
retards de paiement étaient financièrement intéressants pour les débiteurs dans la plupart des Etats membres en raison du faible niveau des intérêts de retard et / ou la lenteur des procédures de recours. Il s'agit de faire en sorte que les conséquences d'un dépassement des délais de paiement soient telles qu'elles découragent cette pratique. La notion de «transaction commerciale" s'entend de manière restrictive comme "toute transaction entre des entreprises ou entre des entreprises et les pouvoirs publics qui conduit à la fourniture de marchandises ou à la prestation de services contre rémunérations » (art 2, point 1), incluant les professions libérales. L'article 5 de cette directive invite les Etats membres à veiller à ce qu'un titre exécutoire puisse être obtenu dans les 90 jours s'il n'y a pas de contestation portant sur la dette ou des points de procédure, sans cependant exiger des Etats qu'ils prennent des mesures spécifiques sur ce point.

\section{3-2-2 L'accès à une justice efficace dans le cadre de la coopération judiciaire}

La levée des obstacles à l'obtention d'un titre exécutoire efficace en matière de litiges transfrontières relève des mesures à prendre dans le domaine de la coopération judiciaire, sur le visa de l'article 65 du Traité. $C^{\prime}$ est la fonction prévue au point c de cet article (« éliminer les obstacles au bon déroulement des procédures civiles, au besoin en favorisant la compatibilité des règles de procédure civile applicables dans les États membres »), qui est la plus importante : elle donne accès au droit procédural interne des Etats membres, avant toute mesure d'exécution, dans des conditions qui tendent de plus en plus à s'affranchir des limites des transactions transfrontières.

\section{Les propositions du Conseil Européen}

Le Conseil européen a développé ces différentes questions lors de sa réunion de Tampere des 15 et 16 octobre 1999, consacrée «à la création d'un espace de liberté, de sécurité et de justice dans l'Union européenne ». Dans le contexte de cette réunion, diverses requêtes étaient adressées au Conseil et à la Commission, sur les litiges transfrontières, et l'exécution des jugements.

En ce qui concerne les litiges transfrontières, il a d'abord été demandé au Conseil d'établir, sur la base de propositions de la Commission, des règles de procédure spéciales communes "en vue de simplifier et d'accélérer le règlement des litiges transfrontaliers concernant les demandes de faible importance en matière civile et commerciale ainsi que les créances alimentaires et les demandes certaines » (paragraphe 30). De plus, un chapitre consacré à la convergence accrue dans le domaine du droit civil invite le Conseil et la Commission à élaborer « de nouvelles dispositions de

119 Economie et Institutions $-n^{\circ} 4-1^{\text {e }}$ semestre 2004 
droit procédural dans les affaires transfrontalières, concernant (...), notamment les mesures provisoires, l'obtention des preuves, l'injonction de payer » (art. 38). On remarquera que dans la première proposition, la catégorie de «demande de faible importance » concerne à la fois la matière civile et commerciale, et renvoie davantage à l'action des créanciers professionnels qu'à celle des consommateurs. Dans la deuxième proposition, l'injonction de payer désigne clairement les demandes des entreprises.

Il a été ensuite demandé à la Commission de faire des propositions en matière de reconnaissance mutuelle des décisions judiciaires. Il s'agit notamment de réduire les mesures intermédiaires nécessaires à la reconnaissance et l'exécution des jugements, visant dans un premier temps « les droits concernant les demandes de faible importance en matière civile ou commerciale et certains litiges relevant du droit de la famille». Ce dispositif pourrait s'accompagner de la fixation de normes minimales pour certains aspects de procédure civile (paragraphe 34 des recommandations). Le paragraphe 37 in fine prévoit également la réalisation de travaux concernant, d'une part le titre exécutoire européen, d'autre part et surtout, « des aspects du droit procédural pour lesquels la fixation de normes minimales communes est considérée comme nécessaire (...), dans le respect des principes fondamentaux du droit des Etats membres ». Là encore, matière civile et commerciale sont étroitement liées, et la partie bénéficiaire des mesures exécutoires au moindre coût est le professionnel plus que le consommateur.

L'ensemble des questions de procédure est à la charge de la Direction Générale Justice et affaires intérieures. Sous son égide, deux actions ont été entreprises dans le domaine de la reconnaissance mutuelle: une proposition de règlement du Conseil portant création d'un titre exécutoire européen pour les créances incontestées ${ }^{37}$; un Livre vert sur une " procédure européenne d'injonction de payer et sur des mesures visant à simplifier et à accélérer le règlement des litiges portant sur des montants de faible importance ${ }^{38}$, élaboré à partir des résultats d'une enquête menée en 2001 auprès des Etats membres ${ }^{39}$.

$37 \operatorname{COM}(2002) 159$ final, $18-04-2002$.

38 COM (2002) 746 final, 20-12-2002.

39 L'enquête a été traitée dans un rapport remis en 2001 à la Commission. E. Serverin, C. Moreau, "Des procédures de traitement judiciaire des demandes de faible importance ou non contestées dans les droits des Etats membres de l'Union européenne", IDHE- Cachan. Les résultats ont été utilisés dans la rédaction du Livre vert.

Economie et Institutions $-n^{\circ} 4-1^{e}$ semestre 2004 


\section{Une simplification pour les créances incontestées}

La proposition de règlement sur le titre exécutoire européen, issu d'une réunion informelle des ministres de la justice qui s'est tenue à Stockholm les 8 et 9 février 2001, constitue le projet pilote de la suppression de l'exequatur. Son motif est d'ordre économique : «le recouvrement rapide des impayés est une nécessité absolue pour le commerce et représente une préoccupation constante des milieux économiques concernés par le bon fonctionnement du marché intérieur » (...), et la procédure "vise à conférer un avantage tangible aux créanciers en leur permettant d'obtenir une exécution rapide et efficace à l'étranger sans intervention des autorités judiciaires de l'Etat membre dans lequel l'exécution est requise avec les retards et les frais que cela suppose. » (pp.2-3). La procédure consiste en une certification d'une décision qui la rend exécutable en tout autre Etat membre sans qu'il soit besoin de recourir à un exequatur. Elle est réservée aux créances incontestées, c'est-à-dire à toutes les situations dans lesquelles un créancier a obtenu un titre constatant une créance pécuniaire sans que le débiteur en ait contesté le principe ni le montant.

\section{Une simplification pour les litiges de faible importance}

Le Livre vert du 20 décembre 2002 concernant « une procédure européenne d'injonction de payer et les mesures visant à simplifier et à accélérer le règlement des litiges portant sur des montants de faible importance ", vient prolonger cette proposition en formulant toute une série de questions sur la possibilité de créer une procédure simplifiée (par voie de règlement ou de directive) pour ces deux situations. Mais alors que le titre exécutoire européen est limité aux litiges transfrontières, l'applicabilité d'un instrument européen aux litiges purement internes est également envisagée, bien que, comme le fait remarquer la Commission, "le principe de reconnaissance mutuelle traite essentiellement des moyens de faciliter la reconnaissance et l'exécution des décisions rendues dans un autre Etat membre et non du rapprochement ou de l'harmonisation du droit procédural ». Autrement dit, le programme n'exclut pas que soient pris des règlements ou des directives pour atteindre le droit interne, même si l'hypothèse prend la forme prudente de questions posées aux destinataires du Livre vert. Si cette extension est envisagée, c'est pour des raisons qui tiennent aux risques concurrentiels que peut présenter la distorsion entre les ordres juridiques. Cette fois, les bénéficiaires de ces projets sont clairement les entreprises. Selon les auteurs du Livre vert, «l'égalité des citoyens et des partenaires économiques au sein d'un espace intégré présuppose une égalité d'accès à l'arsenal juridique. (...) Une entreprise opérant dans un Etat membre dont le système judiciaire assure une exécution rapide et efficace des demandes pourrait jouir d'un atout compétitif substantiel par rapport à

121 Economie et Institutions $-n^{\circ} 4-1^{\text {e }}$ semestre 2004 
une entreprise exerçant ses activités dans un environnement juridique dépourvu de voies de recours d'une efficacité comparable (...), ce qui pourrait aboutir à ce que les entreprises soient dissuadées de mettre en pratique le droit à la liberté d'établissement dans les autres Etats membres » (p. 7).

Le questionnaire présenté par la Commission détaille tous les aspects des procédures pertinents pour répondre efficacement à la demande économique des usagers des tribunaux. S'agissant de l'injonction de payer, le Livre vert relève que si tous les Etats membres mettent en oeuvre des procédures spécifiques pour le recouvrement, « une situation impliquant un déséquilibre du marché au niveau de l'efficacité des outils procéduraux mis à disposition des créanciers dans les différents Etats membres s'apparente à une distorsion de concurrence au sein du marché intérieur ». L'affirmation s'appuie sur une enquête citée dans une communication de la Commission en 1998, selon laquelle « la proportion de retards de paiement intentionnels est largement inférieure à la moyenne communautaire dans les Etats membres où l'obtention et l'exécution des décisions sont rapides, peu coûteuses et efficaces » (p. 12, note 15).

Dans le droit fil de ces travaux, la Commission européenne a présenté le 19 mars 2004 une proposition de règlement du Parlement européen et du Conseil instituant une procédure européenne d'injonction de payer (COM / 2004/0173 final-COD 2004/0055). Cette proposition s'oriente vers la mise en place d'un instrument juridique étendu considérant que " dans le contexte particulier de la procédure de recouvrement de créances incontestées, sa limitation aux situations transfrontalières aurait des conséquences politiques et économiques fâcheuses ». L'argumentaire développé est strictement économique, et s'adresse directement aux entreprises, à qui est garanti l'équilibre des prestations judiciaires dans les différents pays ${ }^{40}$. On ne peut dire plus clairement que le tribunal est un

40 Point 2-2 de l'exposé des motifs : "En premier lieu, l'accès des opérateurs économiques à des mécanismes présentant des niveaux d'efficacité très différents entraîne une distorsion de la concurrence au sein du marché intérieur, que les acteurs soient domiciliés dans des États membres différents ou dans le même État membre. Deux entreprises se livrant concurrence dans un État membre, mais dont une est domiciliée dans ce même État membre, ne sont pas sur un pied d'égalité si seule celle qui est domiciliée à l'étranger peut recourir à une procédure européenne d'injonction de payer efficace. De même, une entreprise dont la plupart des clients se trouvent à l'étranger pourrait retirer un avantage non négligeable de l'existence d'une telle procédure, par rapport à un concurrent domicilié dans le même État membre, mais qui fait la plupart de ses affaires à l'intérieur du pays. De plus, et notamment dans le cas des États membres qui n'offrent pas à l'heure actuelle de mécanisme très efficace de recouvrement de créances non contestées, il sera politiquement très difficile d'expliquer aux créanciers comme aux débiteurs pourquoi le mécanisme

Economie et Institutions $-\mathrm{n}^{\circ} 4-1^{\mathrm{e}}$ semestre 2004 
prestataire de services, qui doit fournir des instruments juridiques efficaces aux opérateurs présents sur le marché.

\section{Conclusion : Penser les tribunaux comme prestataires de service}

Dès lors que le tribunal est vu comme un prestataire de service, il reste à déterminer le coût, et la qualité de la prestation fournie, tant pour l'usager que pour le citoyen. En effet, le maintien d'un service public de la justice n'entraîne pas seulement un coût pour les usagers (notamment lorsque le recours à un avocat est obligatoire), mais engage une dépense publique à laquelle tous contribuent, et qui nécessite de procéder à des choix.

Du côté des usagers, la question se pose du coût d'accès au service de la justice, et la réponse ne peut avoir un caractère général, en raison de la diversité des prestations attendues: les consommateurs attendent que les biens soient livrés, les victimes que les préjudices soient compensés, les professionnels que le prix soit payé, les créanciers d'aliments que les pensions soient fixées. Chaque demandeur est conduit à établir une balance coût /avantage, dans laquelle interviennent des variable comme le degré de nécessité de la procédure (on ne peut divorcer, adopter, changer de régime matrimonial... sans juge), la valeur de la prestation (à comparer au coût du procès), son degré de certitude (qui dépend des preuves disponibles), sa fréquence (demande occasionnelle, ou répétitive).

Du côté des Etats, les arguments économiques ne manquent pas pour soutenir que la justice est, et doit rester, un bien rare. Mais les incidences économiques de cette rareté sur le soutien que les Etats doivent apporter à la garantie de l'exécution des contrats sont également à prendre au sérieux. Loin d'orienter, comme le veut une certaine mode contemporaine, vers la recherche de procédés d'évitement du tribunal, les textes internes, conventionnels, et communautaires poussent de manière continue le système de justice vers plus d'accessibilité et d'efficacité. Qu'ils le veuillent ou non, les Etats sont contraints à investir dans leur appareil de justice, sans

prévu dans les situations transfrontalières est plus efficace que celui qui existe sur le plan interne. La grande majorité des observations formulées en réaction au Livre vert par des opérateurs économiques ou des organisations les représentant, de même que l'avis du Comité économique et social européen, confirment la demande d'une procédure européenne d'injonction de payer universellement applicable, sans différenciation entre les affaires internes et les affaires transfrontalières".

123 Economie et Institutions $-n^{\circ} 4-1^{e}$ semestre 2004 
pouvoir ni déléguer cette mission régalienne, ni imposer aux usagers le recours à un arbitrage, justice privée qui ne peut être établie qu'avec l'accord des parties.

Cette orientation prend à contre-pied les choix théoriques et empiriques d'une partie importante de la recherche (sociologique, économique, juridique), qui s'est détournée de l'étude (empirique), des contentieux pour privilégier une approche (abstraite) des règlements amiables. Elle s'oppose également aux propositions de réforme sur la justice, qui restent marquées par une vision pathologique du procès ${ }^{41}$. Le monde politique comme le monde académique sont bien loin d'avoir pris toute la mesure de la dimension économique de l'appareil juridictionnel ordinaire.

\section{Références}

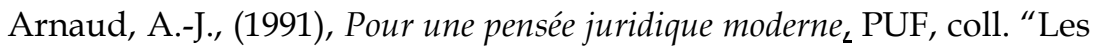
voies du droit".

Barthélémy, J., (1899), Essai d'une théorie sur les droits subjectifs, Paris

Bazzoli, L. et Th. Kirat (1997), «Deux regards non hayékiens sur l'efficience et la sélection des règles juridiques: l'économie

41 Cette attitude est très répandue dans les discours officiels sur la justice. Un exemple récent et caricatural est fourni par l'avis remis le 18 mars 2004 par le Conseil économique et social, sur le rapport présenté par J-P. Noury, intitulé "La judiciarisation de l'économie ". En prenant comme acquis un processus de "judiciarisation " dont aucune preuve n'est jamais apportée, ce rapport se présente comme un vaste florilège de lieux communs sur la justice, assorti de propositions qui prônent tout et son contraire. Il est dit ainsi que le droit à agir doit être " facilité " (p.8), et affirmé de l'autre que les constitutions de partie civile, présentées comme "l'utilisation du service public au profit d'intérêts privés" (!), doivent être étroitement contrôlées (p.17). Les magistrats ne sont pas en reste, qui ne manquent pas de véhiculer une vision défiante des usagers de la justice. Deux exemples récents attestent de la persistance de cette idéologie. Un rapport remis sur la "qualité de la justice " évoque "l'obsession procédurale ", des "plaignants d'habitude ", et se refuse à utiliser la formule "d'usager de la justice ". (La qualité de la justice, Marie-Luce Cavrois, Hubert Dalle et Jean-Paul Jean, coll. Perspectives sur la justice, La Documentation Française, Paris, 2002, p. 25). Un autre rapport intitulé "Célérité et qualité de la Justice " considère que le juge doit "veiller "à ce que la justice ne soit pas abusivement encombrée par le fait de justiciables procéduriers - voire par des quérulents processifs - au détriment de tous ceux qui demandent à être restaurés dans leurs droits ou qui prétendent à une solution qu'ils estiment juste et équitable ". Ce rapport développe des propositions très voisines de celles du CES, en faisant état de "l'impérieuse nécessité de contenir les plaintes avec constitution de partie civile " (Rapport J-Claude Magendie, Président du TGI de Paris, 15 juin 2004, p.24 et 114).

Economie et Institutions $-n^{\circ} 4-1^{e}$ semestre 2004 
institutionnaliste et l'analyse économique du droit», Archives de philosophie du droit, t. 42, pp. 201-227.

Blankenburg, E. (1994a), “La mobilisation du droit. Les conditions du recours et du non recours à la justice", Droit et Société, 28, p. 691-703.

Blankenburg, E. (1994b), "The Infrastructure for Avoiding Civil Litigation: Comparing Cultures of Legal Behavior in The Netherlands and West Germany", Law \& Society Review, vol. 28, n 4, pp. 789-808.

Bonafé-Schmitt, J-P. (1992), La médiation, une justice douce, Syros Alternative.

Bonnard, G. Précis de droit administratif, Paris, 3ème éd.

Burbank, J., (2003), « Litiges civils et société dans la Russie rurale des années 1905-1917 », Genèses, Belin, n50, mars, pp.24-49.

Cappelletti M, R. David, (1984), Accès à la justice et Etat Providence, Economica.

Cavrois, M-L., H. Dalle, J.-P. Jean, (2002), La qualité de la justice, ENM, Mission de recherche «Droit et justice », la Documentation française.

Coase, R. (1959), «The Federal Communications Commission», The Journal of Law and Economics, octobre, pp. 1-40.

Coase, R. (1960), «The Problem of Social Cost», The Journal of Law and Economics, oct., vol. III, pp 1-44.

Coase, R. (1988), The Firm, the Market and the Law, University of Chicago Press. Traduction française par André Duval et Boualem Aliouat: La firme, le marché et le droit, Diderot éditeur, 1997.

Brisset, "Quelques perspectives historiques sur la règle «Nul ne peut se faire justice à lui-même », Arch. Phil. Dr. 1976, p. 205.

Commons, J.R., (1931), «Institutional Economics», American Economic Review, p. 648-657.

Commons, J.R., (1938), « The problem of correlating law, economics, and ethics ", dans Recueil d'études sur les sources du droit en l'honneur de François Gény, Librairie du recueil Sirey, t.3, pp. 124-144.

Demolombe, (1855-1866), Cours de code Napoléon, Paris, t. IX, n³38.

Doriat-Duban, M., (2001), "Alternative Dispute resolution in the french Legal System : an empirical study ", dans Law and Economics in civil Law Countries, B. Deffains et Th. Kirat eds, JAI, Elsevier Science, pp 183-199.

Durkheim, E., (1978) [1897]), De la division du travail social, Puf, 10ème édition.

Favoreu, L., (1964), Du déni de justice en droit public français, Paris, LGDJ.

Felstiner, W., R. Abel, A. Sarat, (1980-1981), «The Emergence and Transformations of Disputes : Naming, Blaming, Claming», Law $\mathcal{E}$ Society Review, 15, 631.

Hart, H.L.A (1976), Le concept de droit, Bruxelles Publication des Facultés universitaires de Saint-Louis, trad. M. Van de Kerchove.

125 Economie et Institutions $-n^{\circ} 4-1^{e}$ semestre 2004 
Hauriou, M. (1919), Précis de droit administratif et de droit public, 9ème édition, Sirey.

Hirsch, W.Z., (1979), Law and Economics. An introductory Analysis, University of California, Academic Press.

Jhering, R., von (1901), L'évolution du droit, Paris, Chevalier-Marescq, trad. de Zweck im Recht, 1886, O. de Meulenaere.

Kelsen, H. (1962), Théorie pure du droit, Paris, Dalloz, trad. Ch. Eisenmann.

Kelsen, H. (2000), «Une théorie réaliste et la théorie pure du droit. Remarques sur 'On Law and justice d'Alf Ross', trad. Sommeregger G. et E. Millard, Annnales de la Faculté de droit de Strasbourg.

Kirat, T. (1999), Economie du droit, Repères, La Découverte.

Kirat, T. et E. Serverin (2000), Dialogue entre droit et économie à propos des règles juridiques et de l'action, in Le droit dans l'action économique, T. Kirat et E. Serverin (dir.) Paris, CNRS Editions, p.5-22.

Landes, W.M., et R.A. Posner, (1976), «Legal Precedent : a theoretical and Empirical Analysis», Journal of Law and Economics, XIX (2).

Landes, W.M., et R.A. Posner, (1979), «Adjudication as a Private Good», Journal of Legal Studies, VIII, (2).

Lange, (1699), Le nouveau praticien français, Paris, Guignard, 8ème édition.

Magendie, J-Cl., (2004), Qualité et célérité de la justice. La gestion du temps dans le procès, Rapport au Garde des sceaux, Ministre de la justice.

Martin, R., (1998), "Un virus dans le système des défenses du nouveau code de procédure civile: le droit d'action", Revue Générale des Procédures, n³, p. 419-426.

Motulsky, H. (1964), "Le droit subjectif et l'action en justice", A.P.D,tome IX, p. 215-230.

Posner, R.A., (1977), Economic Analysis of Law, Boston, Little, Brown.

Ripert, G., (1951), Aspects juridiques du capitalisme moderne, 2ème édition Paris, LGDJ.

Ross, A. (1957), « What is justice? Compte rendu de l'ouvrage de Hans Kelsen », California Law Review, 45, P. 564 et s.

Roubier, P., (1964), «Délimitation et intérêts pratiques de la catégorie des droits subjectifs », A.P.D., tome IX, p. 83-96.

Rouchaud-Joet, A-M., (2003), «L'amorce d'un droit procédural européen. Présentation de l'espace européen en matière civile ", dans G. Deleval et J. Hubin eds.,Espace judiciaire et social européen, Actes du colloque des 5 et 6 novembre 2001, De Boeck et Larcier, Bruxelles.

Seizelet, E., (2002), Justice et magistrature au Japon, PUF, coll. Droit et justice. 
Serverin, E., (2001), "The negotiation of disputed rights or how the Law comes to Economics", dans Law and Economics in civil Law Countries, B. Deffains et Th. Kirat (eds), JAI, Elsevier Science pp.43-60, Serverin, E., (2002) «Quels faits sociaux pour une science empirique du droit? ", Droit et Société n50, LGDJ, pp.59-68.

Storme, M., (1995), Rapprochement du droit judiciaire de l'Union européenne- Approximation of judiciary law in the European Union, Dordrecht/Boston/Londres.

Stanziani, A., (2003), "Action économique et contentieux judiciaires. Le cas du plâtrage des vins en France, 1851-1905 », Genèses, 50, pp.7190.

Weber, M., (1965), Essai sur la théorie de la science, Paris, Plon, trad. J. Freund.

Weber, M., (1995), "Economie et société", T1, Les catégories de la sociologie, T2, L'organisation et les puissances de la société dans leurs rapports avec l'économie ", Plon, Pocket.

Weber, M., (1986) Sociologie du droit, PUF, Coll. Recherches politiques. trad. J. Grosclaude, traduction de l'édition Winckelmann, 1960.

Weber, M. (2001), Rudolf Stammler et le matérialisme historique, Presses de l'Université de Laval, Cerf, trad. M. Coutu et Dominique Leydet.

Wiederkher, G., (1981), «La notion d'action en justice selon l'article 30 du nouveau code de procédure civile », Mélanges Hébraud, Toulouse 1981, p. 951.

127 Economie et Institutions $-\mathrm{n}^{\circ} 4-1^{\mathrm{e}}$ semestre 2004 